\title{
Surfaces in three-dimensional Lie groups *
}

\author{
Dmitry A. Berdinsky ${ }^{\dagger} \quad$ Iskander A. Taimanov ${ }^{\ddagger}$
}

\section{Introduction}

In the present paper we extend the methods of the Weierstrass (or spinor) representation of surfaces in $\mathbb{R}^{3}\left[10,11\right.$ and $S U(2)=S^{3}$ 12 for surfaces in the three-dimensional Lie groups Nil,$\widetilde{S L}_{2}$, and Sol endowed with the so-called Thurston's geometries 9 .

The main feature of this approach is that the geometry of a surface is related to the spectral properties of the corresponding Dirac operator. Therewith this approach reveals some unknown before geometric meanings of the Willmore functional and the Willmore conjecture which states that for tori the Willmore functional attains its minimum on the Clifford torus.

A function $\psi$ generates a surface in $\mathbb{R}^{3}$ via the Weierstrass formulas if and only if it meets some equation of the Dirac type where the Dirac operator in general has two potentials $U$ and $V$ which coincide for the case of surfaces in $\mathbb{R}^{3}$. Given a surface $M$ in $\mathbb{R}^{3}$, the integral

$$
E(M)=\int_{M} U V \frac{i d z \wedge d \bar{z}}{2}
$$

equals to $\frac{1}{4} \int_{M} H^{2} d \mu$ where $H$ is the mean curvature and $d \mu$ is the induced area form on $M$, i.e. $E(M)$ is equal up to a multiple to the Willmore functional

$$
\mathcal{W}(M)=\int_{M} H^{2} d \mu
$$

(see [10]).

The same was established for surfaces in $S U(2)$ in 12 although the Weierstrass formulas have to be replaced by an analogous construction valid for noncommutative Lie groups.

Looking for a physical confirmation of the Willmore conjecture we notice that for the Clifford torus the spectral curve which was defined for general tori

\footnotetext{
${ }^{*}$ The work is supported by RFBR (grant no. 03-01-00403) and by the programm of fundamental researches of RAS "Mathematical methods in nonlinear dynamics".

${ }^{\dagger}$ Department of Mechanics and Mathematics, Novosibirsk State University, 630090 Novosibirsk, Russia; e-mail: berdinsky@ngs.ru .

‡Institute of Mathematics, 630090 Novosibirsk, Russia; e-mail: taimanov@math.nsc.ru.
} 
in 11] has zero geometric genus. Moreover the deviation of the spectral curve of a torus from the flat curve which is the spectral curve of the Dirac operator with vanishing potentials $U=V=0$ is measured by $E(M)$. This led us to an approach for proving the Willmore conjecture by proving that in each conformal class the minimum of the Willmore functional is attained on a torus with the minimal geometric genus (this part some time ago was announced to be proved by M.U. Schmidt (see math.DG/0203224 in http://arxiv.org)) as the first step and by checking the conjecture for tori with the minimal geometric genus in their conformal classes as the final step.

Recently M. Haskins proposed to treat the genus of the spectral curve as a measure of geometric complexity for some other variational problems of geometry [6].

In the present paper we derive the analogous formulas for the functional $E(M)$ for surfaces in Nil, $\widetilde{S L}_{2}$, and Sol. It appears it has very nonexpectable geometric properties. We call it the spinor energy or just the energy of a surface.

Until now we even do not know is it bounded from below or not. For surfaces in Sol we do not prove that it is real-valued. However it measures the deviation of the spectral curve of a torus from the flat curve and we think that that is enough for confirming its geometric importance.

The problems of finding the analogs of the Willmore conjecture for such functionals and describing their extremals are very interesting.

In the present paper we also derive the equations for $\psi$ corresponding to minimal surfaces in Lie groups and by our means obtain another proof to Abresch's result that for constant mean curvature surfaces in Nil and $\widetilde{S L}{ }_{2}$ certain quadratic differentials are holomorphic [1]. It would be very intriguing to relate these conditions to integrable systems as it was done for constant mean curvature surfaces in $\mathbb{R}^{3}$ and $S^{3}$.

We note that the approach by Abresch differs from our and bases on a representation of Nil and Sol as line bundles of constant curvature over surfaces of constant curvature (see also the recent paper 3] where this property is used for studying surfaces Nil and Sol). It looks that the failure of such a representation for Sol explains why our approach does not lead to such satisfactory understanding of surfaces as in the cases of Nil and $\widetilde{S L}$.

We notice that the study of integrable surfaces in arbitrary Lie groups by methods of integrable systems was first attempted in 5 . We also hope that our approach will be helpful in studying global properties of minimal and constant mean curvature surfaces in Lie groups in the spirit of [2, 4].

The results of this paper were partially exposed on the conference on the surface theory in Benediktbeuern (January 2005).

We thank U. Abresch for many helpful conversations and an explanation of his result on holomorphic quadratic differentials for constant mean curvature surfaces in Nil and Sol. 


\section{Preliminary facts}

\subsection{Left invariant metrics on Lie groups}

Recall that a metric $\langle\xi, \eta\rangle$ on Lie group $G$ is called left invariant if it is invariant with respect to left translations:

$$
L_{g}: G \stackrel{\times g}{\longrightarrow} G \quad: \quad h \rightarrow g h, h \in G,
$$

i.e. for all vectors $\xi$ and $\eta$ tangent to $G$ at $h$, the inner product of their translations by $g$, and the inner product of $\xi$ and $\eta$ coincide:

$$
\langle\xi, \eta\rangle=\left\langle L_{g}^{*} \xi, L_{g}^{*} \eta\right\rangle, \quad \xi, \eta \in T_{h} G, g, h \in G .
$$

Clearly every left invariant metric is determined by the inner product of vectors tangent at the unit $1 \in G$ of the group $G$, i.e. by the inner product on the Lie algebra $\mathcal{G}$ of $G$.

For calculating the Levi-Civita connection we apply the general tetrad formalism known from mathematical physics which is as follows.

Given vector fields $e_{1}, \ldots, e_{n}$ on an $n$-dimensional manifold $M$ such that at every point the corresponding vectors form an orthonormal base:

$$
\left\langle e_{i}, e_{j}\right\rangle=\delta_{i j}, \quad i, j=1, \ldots, n,
$$

the Levi-Civita connection is given by the following formulas:

$$
\begin{gathered}
\Gamma_{j k}^{i}=\frac{1}{2}\left(c_{k j}^{i}+c_{i k}^{j}+c_{i j}^{k}\right), \\
\nabla_{e_{k}} e_{j}=\Gamma_{j k}^{i} e_{i},
\end{gathered}
$$

where

$$
\left[e_{i}, e_{j}\right]=c_{i j}^{k} e_{k} .
$$

In our case let us take an orthonormal base $\xi_{1}, \ldots, \xi_{n}$ for the tangent space at the unit of the group $G$ and extend it to vector fields on the whole group by left translations:

$$
e_{i}(g)=L_{g}^{*} \xi_{i}, \quad i=1, \ldots, n, g \in G .
$$

Then $c_{j k}^{i}$ are just the structure constants of the Lie algebra $\mathcal{G}$ of the group $G$ and putting for simplicity

$$
\alpha_{i j k}=\left\langle\left[e_{i}, e_{j}\right], e_{k}\right\rangle=c_{i j}^{k}
$$

we derive

$$
\nabla_{e_{k}} e_{j}=\frac{1}{2} \sum_{i}\left(\alpha_{k j i}+\alpha_{i k j}+\alpha_{i j k}\right) e_{i}
$$

In particular, $\alpha_{i j k}$ is a skew-symmetric tensor for a compact Lie group $G$ with the Killing metric and the formula (1) reduces to

$$
\nabla_{X} Y=\frac{1}{2}[X, Y]
$$

where $X$ and $Y$ are left invariant vector fields. For details we refer to 8$]$. 


\subsection{The derivational equations}

Let $\Sigma$ be a surface immersed into $G$ and let

$$
f: \Sigma \rightarrow G
$$

be the immersion. Choose a conformal parameter $z=x+i y$ on $\Sigma$ (or, more precisely, in a domain of $\Sigma$ ) and denote by $\mathbf{I}=e^{2 \alpha} d z d \bar{z}$ the induced metric.

Let us consider the pullback of $T G$ to a $\mathcal{G}$-bundle over $\Sigma: \mathcal{G} \rightarrow E=$ $f^{-1}(T G) \stackrel{\pi}{\rightarrow} \Sigma$ and the differential

$$
d_{\mathcal{A}}: \Omega^{1}(\Sigma ; E) \rightarrow \Omega^{2}(\Sigma ; E),
$$

which acts on $E$-valued 1-forms as follows. Let us write down a form $\omega$ as

$$
\omega=u d z+u^{*} d \bar{z} .
$$

Then

$$
d_{\mathcal{A}} \omega=d_{\mathcal{A}}^{\prime} \omega+d_{\mathcal{A}}^{\prime \prime} \omega
$$

where

$$
d_{\mathcal{A}}^{\prime} \omega=-\nabla_{\bar{\partial} f} u d z \wedge d \bar{z}, \quad d_{\mathcal{A}}^{\prime \prime} \omega=\nabla_{\partial f} u^{*} d z \wedge d \bar{z} .
$$

By straightforward computations we obtain the first derivational equation

$$
d_{\mathcal{A}}(d f)=0 .
$$

By the definition of the tension vector $\tau(f)$, we have

$$
d_{\mathcal{A}}(* d f)=f \cdot\left(e^{2 \alpha} \tau(f)\right) d x \wedge d y=\frac{i}{2} f \cdot\left(e^{2 \alpha} \tau(f)\right) d z \wedge d \bar{z}
$$

where $f \cdot \tau(f)=2 H N, N$ is the normal vector and $H$ is the mean curvature. Hence we obtain the second derivational equation:

$$
d_{\mathcal{A}}(* d f)=i e^{2 \alpha} H N d z \wedge d \bar{z} .
$$

For $S=S U(2)$ we expose this scheme in [12] (see also the derivation of the harmonicity equation for surfaces in Lie groups in [7]).

Since the metric is left invariant we write down these equations in terms of

$$
\Psi=f^{-1} \partial f, \quad \Psi^{*}=f^{-1} \bar{\partial} f
$$

as

$$
\begin{gathered}
\partial \Psi^{*}-\bar{\partial} \Psi+\nabla_{\Psi} \Psi^{*}-\nabla_{\Psi^{*}} \Psi=0, \\
\partial \Psi^{*}+\bar{\partial} \Psi+\nabla_{\Psi} \Psi^{*}+\nabla_{\Psi^{*}} \Psi=e^{2 \alpha} H f^{-1}(N) .
\end{gathered}
$$

The equation (4) is equivalent to (2) and the equation (5) is equivalent to (3). 
In the sequel we assume that the Lie group $G$ is three-dimensional and we choose an orthonormal basis $e_{1}, e_{2}, e_{3}$ for the inner product on the Lie algebra $\mathcal{G}$ of the Lie group $G$.

Let us expand $\Psi$ and $\Psi^{*}$ in this basis as

$$
\Psi=\sum_{k=1}^{3} Z_{k} e_{k}, \quad \Psi^{*}=\sum_{k=1}^{3} \bar{Z}_{k} e_{k}
$$

and rewrite (44) and (5) in terms of $Z_{k}, k=1,2,3$, as follows

$$
\begin{gathered}
\sum_{j}\left(\partial \bar{Z}_{j}-\bar{\partial} Z_{j}\right) e_{j}+\sum_{j, k}\left(Z_{j} \bar{Z}_{k}-\bar{Z}_{j} Z_{k}\right) \nabla_{e_{j}} e_{k}=0, \\
\sum_{j}\left(\partial \bar{Z}_{j}+\bar{\partial} Z_{j}\right) e_{j}+\sum_{j, k}\left(Z_{j} \bar{Z}_{k}+\bar{Z}_{j} Z_{k}\right) \nabla_{e_{j}} e_{k}= \\
2 i H\left[\left(\bar{Z}_{2} Z_{3}-Z_{2} \bar{Z}_{3}\right) e_{1}+\left(\bar{Z}_{3} Z_{1}-Z_{3} \bar{Z}_{1}\right) e_{2}+\left(\bar{Z}_{1} Z_{2}-Z_{1} \bar{Z}_{2}\right) e_{3}\right] .
\end{gathered}
$$

Here we assumed that the basis $\left\{e_{1}, e_{2}, e_{3}\right\}$ is positively oriented and therefore

$$
f^{-1}(N)=2 i e^{-2 \alpha}\left[\left(\bar{Z}_{2} Z_{3}-Z_{2} \bar{Z}_{3}\right) e_{1}+\left(\bar{Z}_{3} Z_{1}-Z_{3} \bar{Z}_{1}\right) e_{2}+\left(\bar{Z}_{1} Z_{2}-Z_{1} \bar{Z}_{2}\right) e_{3}\right]
$$

(for $G=S U(2)$ with the Killing metric this formula takes the form $f^{-1}(N)=$ $2 i e^{-2 \alpha}\left[\Psi^{*}, \Psi\right] .{ }^{1}$ Since the parameter $z$ is conformal we have

$$
\langle\Psi, \Psi\rangle=\left\langle\Psi^{*}, \Psi^{*}\right\rangle=0, \quad\left\langle\Psi, \Psi^{*}\right\rangle=\frac{1}{2} e^{2 \alpha}
$$

which is rewritten as

$$
Z_{1}^{2}+Z_{2}^{2}+Z_{3}^{2}=0, \quad\left|Z_{1}\right|^{2}+\left|Z_{2}\right|^{2}+\left|Z_{3}\right|^{2}=\frac{1}{2} e^{2 \alpha} .
$$

The first equality implies that the vector $Z$ can be parameterized in the form

$$
Z_{1}=\frac{i}{2}\left(\bar{\psi}_{2}^{2}+\psi_{1}^{2}\right), \quad Z_{2}=\frac{1}{2}\left(\bar{\psi}_{2}^{2}-\psi_{1}^{2}\right), \quad Z_{3}=\psi_{1} \bar{\psi}_{2} .
$$

\subsection{The Dirac operator and the energy of a surface}

The Weierstrass representation is derived by inserting $\psi$ into (6) and (7) (see [10, 12] for such representations of surfaces in $\mathbb{R}^{3}$ and $S^{3}$ ).

In this event the derivational equations takes the form of the Dirac equation

$$
\mathcal{D} \psi=\left[\left(\begin{array}{cc}
0 & \partial \\
-\bar{\partial} & 0
\end{array}\right)+\left(\begin{array}{cc}
U & 0 \\
0 & V
\end{array}\right)\right] \psi=0
$$

\footnotetext{
${ }^{1}$ In $[2]$ the term of 2 in the right hand side of the same formula for $N$ (the formula (47)) was skipped however it was counted in all computations and this misprint does not affect any result.
} 
which is satisfied by

$$
\psi=\left(\begin{array}{c}
\psi_{1} \\
\psi_{2}
\end{array}\right) .
$$

The first fundamental form is equal to

$$
\mathrm{I}=e^{2 \alpha} d z d \bar{z}=\left(\left|\psi_{1}\right|^{2}+\left|\psi_{2}\right|^{2}\right)^{2} d z d \bar{z}
$$

and the left translation of the normal vector is

$$
\begin{gathered}
f^{-1}(N)=e^{-\alpha}\left[i\left(\psi_{1} \psi_{2}-\bar{\psi}_{1} \bar{\psi}_{2}\right) e_{1}-\right. \\
\left.\left.\left(\psi_{1} \psi_{2}+\bar{\psi}_{1} \bar{\psi}_{2}\right) e_{2}+\left(\left|\psi_{2}\right|^{2}-\left|\psi_{1}\right|^{2}\right) e_{3}\right)\right] .
\end{gathered}
$$

Since $\Psi d z=\left(\sum Z_{k} e_{k}\right) d z$ is a correctly defined 1-form, we conclude that the 1-forms

$$
\psi_{1}^{2} d z, \quad \bar{\psi}_{2}^{2} d z, \quad \psi_{1} \bar{\psi}_{2} d z
$$

are globally defined on the whole surface. By the Dirac equation, this implies that the potentials $U$ and $V$ are the squares of $d z d \bar{z}$ forms and therefore

$$
U V d z \wedge d \bar{z}
$$

is a well-defined 2-form on the surface (see [10] for details).

For a surface $M$ immersed into $\mathbb{R}^{3}$ the potentials of the Dirac operator take real values and coincide: $U=V$. For such a surface the following equality holds:

$$
E(M)=\int_{M} U V \frac{i d z \wedge d \bar{z}}{2}=\frac{1}{4} \int_{M} H^{2} d \mu
$$

where $d \mu=e^{2 \alpha} d x \wedge d y$ is the induced measure on the surface and $H$ is the mean curvature. We recall that the Willmore functional equals

$$
\mathcal{W}(M)=\int_{M} H^{2} d \mu
$$

This observation from [10] was the starting point for treating the spectral quantities of this operator $\mathcal{D}$ as the geometric quantities and for physical explanation of the Willmore conjecture by using the spectral curves of immersed tori (see [10, 12]).

Generically $U$ and $V$ do not coincide necessarily and we have to consider the energy of a closed surface as

$$
E(M)=\int_{M} U V d x \wedge d y=\int_{M} U V \frac{i d z \wedge d \bar{z}}{2} .
$$

It is even not clear that it always be real-valued. We shall check that for some special cases. For that we shall use the following simple proposition which follows from the Dirac equation. Indeed we have

$$
\bar{\partial} \psi_{1}=(\operatorname{Re} V+i \operatorname{Im} V) \psi_{2}, \quad \bar{\partial} \bar{\psi}_{2}=(-\operatorname{Re} U+i \operatorname{Im} U) \psi_{1}
$$

which implies 
Proposition 1 Given $\psi$ meeting the Dirac equation (9), the following identity holds

$$
\bar{\partial}\left(\psi_{1} \bar{\psi}_{2}\right)=\left(-\operatorname{Re} U\left|\psi_{1}\right|^{2}+\operatorname{Re} V\left|\psi_{2}^{2}\right|\right)+i\left(\operatorname{Im} U\left|\psi_{1}\right|^{2}+\operatorname{Im} V\left|\psi_{2}\right|^{2}\right) .
$$

\subsection{Thurston's geometries on Lie groups Nil, Sol and $\widetilde{S L}_{2}$}

By Thurston's theorem all three-dimensional maximal simply connected geometries $(X$, Isom $X)$ admitting compact quotient geometries belong to the following list:

1) three geometries with constant sectional curvature: $X=\mathbb{R}^{3}, S^{3}$, or $H^{3}$;

2) a pair of product geometries: $X=S^{2} \times \mathbb{R}$ or $H^{2} \times \mathbb{R}$;

3 ) three geometries modeled on the Lie groups Nil, Sol, and $\widetilde{S L}_{2}$ with certain left invariant metrics.

We refer for details of this classification theorem and for the related famous Thurston geometrization conjecture for three-manifolds to [13, 9].

In the paper we study surfaces in these geometries modelled on noncompact Lie groups Nil, Sol, and $\widetilde{S L}_{2}$. Before going to the surface theory we briefly recall main facts about these geometries referring for an advanced exposition to 9].

\subsubsection{The group Nil}

This group is formed by all matrices of the form

$$
\left(\begin{array}{lll}
1 & x & z \\
0 & 1 & y \\
0 & 0 & 1
\end{array}\right), \quad x, y, z \in \mathbb{R}
$$

with the usual multiplication rule and the left invariant metric

$$
d s^{2}=d x^{2}+d y^{2}+(d z-x d y)^{2} .
$$

The Lie algebra is generated by the elements

$$
e_{1}=\left(\begin{array}{lll}
0 & 1 & 0 \\
0 & 0 & 0 \\
0 & 0 & 0
\end{array}\right), \quad e_{2}=\left(\begin{array}{lll}
0 & 0 & 0 \\
0 & 0 & 1 \\
0 & 0 & 0
\end{array}\right), \quad e_{3}=\left(\begin{array}{lll}
0 & 0 & 1 \\
0 & 0 & 0 \\
0 & 0 & 0
\end{array}\right),
$$

which meet the following commutation relations

$$
\left[e_{1}, e_{2}\right]=e_{3}, \quad\left[e_{1}, e_{3}\right]=\left[e_{2}, e_{3}\right]=0 .
$$

We derive from (1) that the connection is as follows:

$$
\begin{gathered}
\nabla_{e_{1}} e_{2}=-\nabla_{e_{2}} e_{1}=\frac{1}{2} e_{3}, \quad \nabla_{e_{1}} e_{3}=\nabla_{e_{3}} e_{1}=-\frac{1}{2} e_{2}, \\
\nabla_{e_{2}} e_{3}=\nabla_{e_{3}} e_{2}=\frac{1}{2} e_{1}, \quad \nabla_{e_{1}} e_{1}=\nabla_{e_{2}} e_{2}=\nabla_{e_{3}} e_{3}=0 .
\end{gathered}
$$




\subsubsection{The group $\widetilde{S L}_{2}$}

The group $G=\widetilde{S L}_{2}$ is the universal covering of the group $S L(2)$ formed by all real $2 \times 2$-matrices with unit determinant. The group $P S L(2)=S L(2) / \pm 1$ is the group of orientation preserving isometries of the hyperbolic plane $H^{2}$ and it is diffeomorphic to the unit tangent bundle $U H^{2}$ of $H^{2}$. Moreover the left invariant metric on $\widetilde{S L}_{2}$ corresponding to one of the Thurston geometries (i.e., with the maximal isometry group) is the pullback under the projection

$$
\widetilde{S L}_{2} \rightarrow S L(2) \rightarrow P S L(2) \approx U H^{2}
$$

of the metric on $U H^{2}$ which is as follows.

Let us realize the hyperbolic plane as the disc $|z|<1$ on the complex plane with the metric

$$
d s^{2}=\frac{4 d z d \bar{z}}{\left(1-|z|^{2}\right)^{2}}
$$

and take on $U H^{2}$ the metric

$$
d l^{2}=\frac{4\left(d z d \bar{z}+d \varphi^{2}\right)}{\left(1-|z|^{2}\right)^{2}}
$$

where $\varphi$ is the angle coordinate on the unit circles in the tangent spaces to $H^{2}$. We identify the generators of the Lie algebra of $\widetilde{S L}_{2}$ with the generators of the following one-parametric subgroups

$$
\begin{gathered}
g_{x}=\left(\begin{array}{cc}
\frac{1}{\sqrt{1-x^{2}}} & \frac{x}{\sqrt{1-x^{2}}} \\
\frac{x}{\sqrt{1-x^{2}}} & \frac{1}{\sqrt{1-x^{2}}}
\end{array}\right), g_{y}=\left(\begin{array}{cc}
\frac{1}{\sqrt{1-y^{2}}} & \frac{i y}{\sqrt{1-y^{2}}} \\
-\frac{i y}{\sqrt{1-y^{2}}} & \frac{1}{\sqrt{1-y^{2}}}
\end{array}\right), \\
g_{\varphi}=\left(\begin{array}{cc}
e^{i \varphi / 2} & 0 \\
0 & e^{-i \varphi / 2}
\end{array}\right)
\end{gathered}
$$

which act on $H^{2}$ by fractional linear transformations

$$
z \rightarrow \frac{a z+b}{c z+d}, \quad\left(\begin{array}{ll}
a & b \\
c & d
\end{array}\right) \in S L(2) .
$$

The generators of these subgroups are

$$
f_{1}=\left(\begin{array}{ll}
0 & 1 \\
1 & 0
\end{array}\right), \quad f_{2}=\left(\begin{array}{cc}
0 & i \\
-i & 0
\end{array}\right), \quad f_{3}=\left(\begin{array}{cc}
\frac{i}{2} & 0 \\
0 & -\frac{i}{2}
\end{array}\right) .
$$

These generators meet the commutation relations

$$
\left[f_{1}, f_{2}\right]=-4 f_{3}, \quad\left[f_{1}, f_{3}\right]=-f_{2}, \quad\left[f_{2}, f_{3}\right]=f_{1} .
$$

The desired metric on $\widetilde{S L}_{2}$ is induced by the inner product

$$
\left\langle f_{j}, f_{k}\right\rangle=4 \delta_{j k}, \quad j, k=1,2,3,
$$


on the Lie algebra of $\widetilde{S L}_{2}$ and, by (1), this implies

$$
\begin{array}{r}
\nabla_{e_{1}} e_{2}=-\nabla_{e_{2}} e_{1}=-e_{3}, \quad \nabla_{e_{1}} e_{3}=e_{2}, \quad \nabla_{e_{3}} e_{1}=\frac{3}{2} e_{2}, \\
\nabla_{e_{2}} e_{3}=-e_{1}, \nabla_{e_{3}} e_{2}=-\frac{3}{2} e_{1}, \quad \nabla_{e_{1}} e_{1}=\nabla_{e_{2}} e_{2}=\nabla_{e_{3}} e_{3}=0,
\end{array}
$$

where

$$
e_{j}=\frac{1}{2} f_{j}, \quad\left\langle e_{j}, e_{k}\right\rangle=\delta_{j k}, \quad j, k=1,2,3
$$

\subsubsection{The group Sol}

This group consists of all matrices of the form

$$
\left(\begin{array}{ccc}
e^{-z} & 0 & x \\
0 & e^{z} & y \\
0 & 0 & 1
\end{array}\right), \quad x, y, z \in \mathbb{R}
$$

with the usual multiplication rule and the left invariant metric

$$
d s^{2}=e^{2 z} d x^{2}+e^{-2 z} d y^{2}+d z^{2} .
$$

The Lie algebra is generated by the elements

$$
e_{1}=\left(\begin{array}{lll}
0 & 0 & 1 \\
0 & 0 & 0 \\
0 & 0 & 0
\end{array}\right), \quad e_{2}=\left(\begin{array}{lll}
0 & 0 & 0 \\
0 & 0 & 1 \\
0 & 0 & 0
\end{array}\right), \quad e_{3}=\left(\begin{array}{ccc}
-1 & 0 & 0 \\
0 & 1 & 0 \\
0 & 0 & 0
\end{array}\right),
$$

which satisfy the commutation relations

$$
\left[e_{1}, e_{2}\right]=0, \quad\left[e_{1}, e_{3}\right]=e_{1}, \quad\left[e_{2}, e_{3}\right]=-e_{2} .
$$

By (11), we conclude that

$$
\begin{array}{rr}
\nabla_{e_{1}} e_{2}=\nabla_{e_{2}} e_{1}=0, \quad \nabla_{e_{1}} e_{3}=e_{1}, & \nabla_{e_{3}} e_{1}=0 \\
\nabla_{e_{2}} e_{3}=-e_{2}, & \nabla_{e_{3}} e_{2}=0 \\
\nabla_{e_{1}} e_{1}=-\nabla_{e_{2}} e_{2}=-e_{3}, & \nabla_{e_{3}} e_{3}=0
\end{array}
$$

\subsection{The curvature tensors of three-dimensional Lie groups}

By (13), (14) and (15) we compute the curvature tensor

$$
\langle R(X, Y) Z, W\rangle=R_{l k j i} W^{l} Z^{k} Y^{j} X^{i}=\left\langle\left(\nabla_{Y} \nabla_{X}-\nabla_{X} \nabla_{Y}+\nabla_{[X, Y]}\right) Z, W\right\rangle
$$

of the groups Nil, $\widetilde{S L}_{2}$, and Sol. We skip all these simple computations presenting only the result that we need. 
Proposition 2 For the groups $\mathrm{Nil}, \widetilde{S L}_{2}$, and Sol if there are three different indices among $i, j, k, l$ then $R_{i j k l}=0$. The other components of the curvature tensor are as follows

$$
R_{1212}=\left\{\begin{array}{ll}
-\frac{3}{4} & \text { for } \mathrm{Nil} \\
-4 & \text { for } \widetilde{S L}_{2}, \\
1 & \text { for } \mathrm{Sol}
\end{array} \quad R_{1313}=R_{2323}= \begin{cases}\frac{1}{4} & \text { for } \mathrm{Nil} \\
1 & \text { for } \widetilde{S L}_{2} \\
-1 & \text { for } \mathrm{Sol}\end{cases}\right.
$$

Recall that if $|X|=|Y|=1$ and these vectors are linearly independent then

$$
K_{X Y}=\langle R(X, Y) X, Y\rangle
$$

equals the sectional curvature of the plane spanned by $X$ and $Y$. This proposition enables us to compute the sectional curvature of every plane.

\section{The Weierstrass representation for surfaces in Lie groups}

\subsection{A construction of a surface from $\psi$}

In this section we derive the compatibility conditions for a vector function $\psi$, i.e. a criterion for $\psi$ to correspond to an immersion of a surface into Nil, $\widetilde{S L_{2}}$, or Sol. Given a function $\psi$ meeting these conditions for a Lie group $G$ from this list we may construct a surface as follows. ${ }^{2}$

Let $\psi$ be defined on a surface $M$ with a complex parameter $z$. Let us pick up a point $P \in M$.

First, we insert $\psi$ into (8) for the components $Z_{1}, Z_{2}, Z_{3}$ of $\Psi=\sum_{k=1}^{3} Z_{k} e_{k}=$ $f^{-1} \partial f$. Second, we solve the following linear equation in the Lie group $G$ :

$$
f_{z}=f \Psi
$$

with a certain initial data $f(P)=g \in G$. We thus obtain the desired surface as the mapping

$$
f: M \rightarrow G .
$$

It follows from the derivation of the compatibility conditions in 2.1 that any surface $f: M \rightarrow G$ is obtained by this construction. The induced metric takes the form

$$
d s^{2}=e^{2 \alpha} d z d \bar{z}, \quad e^{\alpha}=\left(\left|\psi_{1}\right|^{2}+\left|\psi_{2}\right|^{2}\right),
$$

and the induced measure on $M$ is

$$
d \mu=e^{2 \alpha} \frac{i d z \wedge d \bar{z}}{2}
$$

\footnotetext{
${ }^{2}$ This generalizes the Weierstrass representation of surfaces in $\mathbb{R}^{3}[11$ and $S U(2)$ [12] for surfaces in these groups.
} 
The Hopf quadratic differential equals

$$
\omega=A d z^{2}, \quad A=\left\langle\nabla_{f_{z}} f_{z}, N\right\rangle .
$$

Its explicit formula in terms of $\psi$ depends on the Lie group and the Levi-Civita connection on this group. Indeed, we have

$$
\begin{array}{r}
A=\left\langle\Psi_{z}, N\right\rangle+\left\langle\sum_{j, k} Z_{j} Z_{k} \nabla_{e_{j}} e_{k}, N\right\rangle= \\
=\left(\bar{\psi}_{2} \partial \psi_{1}-\psi_{1} \partial \bar{\psi}_{2}\right)+\left\langle\sum_{j, k} Z_{j} Z_{k} \nabla_{e_{j}} e_{k}, N\right\rangle .
\end{array}
$$

The compatibility conditions takes the form of the Dirac equation (9) and describe $\bar{\partial} \psi_{1}$ and $\partial \psi_{2}$ :

$$
\mathcal{D} \psi=\left[\left(\begin{array}{cc}
0 & \partial \\
-\bar{\partial} & 0
\end{array}\right)+\left(\begin{array}{cc}
U & 0 \\
0 & V
\end{array}\right)\right] \psi=0 .
$$

Another derivatives which are $\partial \psi_{1}$ and $\bar{\partial} \psi_{2}$ are derived as follows. We differentiate $e^{\alpha}$ and obtain

$$
\alpha_{z} e^{\alpha}=\bar{\psi}_{1} \partial \psi_{1}+\psi_{2} \partial \bar{\psi}_{2}+\left(\psi_{1} \partial \bar{\psi}_{1}+\bar{\psi}_{2} \partial \psi_{2}\right),
$$

where, by the Dirac equation, the expression in brackets is written as

$$
\psi_{1} \partial \bar{\psi}_{1}+\bar{\psi}_{2} \partial \psi_{2}=(\bar{V}-U) \psi_{1} \bar{\psi}_{2} .
$$

Together with the formula for the Hopf differential this gives a system for $\partial \psi_{1}$ and $\partial \bar{\psi}_{2}$ :

$$
\left(\begin{array}{cc}
\bar{\psi}_{1} & \psi_{1} \\
\bar{\psi}_{2} & -\psi_{1}
\end{array}\right)\left(\begin{array}{c}
\partial \psi_{1} \\
\partial \bar{\psi}_{2}
\end{array}\right)=\left(\begin{array}{c}
\alpha_{z} e^{\alpha}+(U-\bar{V}) \psi_{1} \bar{\psi}_{2} \\
A-\left\langle\sum_{j, k} Z_{j} Z_{k} \nabla_{e_{j}} e_{k}, N\right\rangle
\end{array}\right)
$$

Resolving this system we obtain the expressions for $\partial \psi_{1}$ and $\bar{\partial} \psi_{2}$ which together with the Dirac equation give us the complete set of the Weingarten equations in terms on $\psi$ :

$$
(\partial-\mathcal{A}) \psi=0, \quad(\bar{\partial}-\mathcal{B}) \psi=0 .
$$

The Codazzi equations are now derived from the zero-curvature conditions

$$
\partial \bar{\partial} \psi_{k}=\bar{\partial} \partial \psi_{k}, \quad k=1,2 .
$$

In the sequel we call $\psi$ the generating spinor of a surface (see [12 for an explanation of this terminology). 


\subsection{The group Nil}

By (13), the derivational equations (6) and (7) take the form

$$
\begin{array}{r}
\partial \bar{Z}_{1}-\bar{\partial} Z_{1}=0, \\
\partial \bar{Z}_{2}-\bar{\partial} Z_{2}=0, \\
\partial \bar{Z}_{3}-\bar{\partial} Z_{3}+\left(Z_{1} \bar{Z}_{2}-\bar{Z}_{1} Z_{2}\right)=0, \\
\partial \bar{Z}_{1}+\bar{\partial} Z_{1}+\left(Z_{2} \bar{Z}_{3}+\bar{Z}_{2} Z_{3}\right)=2 i H\left(\bar{Z}_{2} Z_{3}-Z_{2} \bar{Z}_{3}\right), \\
\partial \bar{Z}_{2}+\bar{\partial} Z_{2}-\left(Z_{1} \bar{Z}_{3}+\bar{Z}_{1} Z_{3}\right)=2 i H\left(\bar{Z}_{3} Z_{1}-Z_{3} \bar{Z}_{1}\right), \\
\partial \bar{Z}_{3}+\bar{\partial} Z_{3}=2 i H\left(\bar{Z}_{1} Z_{2}-Z_{1} \bar{Z}_{2}\right) .
\end{array}
$$

Inserting (8) into these formulas we rewrite the first pair of equations as

$$
\partial \psi_{2}^{2}+\bar{\partial} \psi_{1}^{2}=0
$$

and the pair consisting of the forth and fifth equations is equivalent to

$$
\partial \psi_{2}^{2}-\bar{\partial} \psi_{1}^{2}+i \psi_{1} \psi_{2}\left(\left|\psi_{2}\right|^{2}-\left|\psi_{1}\right|^{2}\right)=-2 H \psi_{1} \psi_{2}\left(\left|\psi_{1}\right|^{2}+\left|\psi_{2}\right|^{2}\right) .
$$

The system of these two equations at the points where $\psi_{1} \psi_{2} \neq 0$ are written in the form of the Dirac equation which is satisfied by continuity on the whole surface:

$$
\begin{gathered}
\mathcal{D}_{\mathrm{Nil}} \psi=\left[\left(\begin{array}{cc}
0 & \partial \\
-\bar{\partial} & 0
\end{array}\right)+\left(\begin{array}{cc}
U_{\mathrm{Nil}} & 0 \\
0 & V_{\mathrm{Nil}}
\end{array}\right)\right] \psi=0, \\
U_{\mathrm{Nil}}=V_{\mathrm{Nil}}=\frac{H}{2}\left(\left|\psi_{1}\right|^{2}+\left|\psi_{2}\right|^{2}\right)+\frac{i}{4}\left(\left|\psi_{2}\right|^{2}-\left|\psi_{1}\right|^{2}\right),
\end{gathered}
$$

where $H$ is the mean curvature of the surface.

REMARK 1 . The tangent plane at a point where $Z_{3}=\psi_{1} \bar{\psi}_{2}=0$ is spanned by the left invariant fields generated by $e_{1}$ and $e_{2}$. Since the commutator of this fields is transversal to their span (i.e., $\left[e_{1}, e_{2}\right]=e_{3}$ ) the left invariant distribution of 2-planes generated by $e_{1}$ and $e_{2}$ is nowhere integrable. Therefore the equality $Z_{3}=0$ can not hold in open subsets of the surface. We indicate that the potentials of the Dirac operator are correctly defined everywhere and therefore the equation $\mathcal{D} \psi=0$ is extended by continuity to the closure of $\left\{Z_{3} \neq 0\right\}$ which, as we argue above, coincides with the whole surface. The same is true for surfaces in $S U(2)$ and $\widetilde{S L}_{2}$ but fails in the case of $G=\mathrm{Sol}$.

The Hopf differential takes the form

$$
A=\left(\bar{\psi}_{2} \partial \psi_{1}-\psi_{1} \partial \bar{\psi}_{2}\right)+i \psi_{1}^{2} \bar{\psi}_{2}^{2}
$$

and the Weingarten equations (17) consists of the Dirac equation and the following system

$$
\begin{gathered}
\partial \psi_{1}=\alpha_{z} \psi_{1}+A e^{-\alpha} \psi_{2}-\frac{i}{2} \psi_{1}^{2} \bar{\psi}_{2}, \\
\bar{\partial} \psi_{2}=-\bar{A} e^{-\alpha} \psi_{1}+\alpha_{\bar{z}} \psi_{2}-\frac{i}{2} \bar{\psi}_{1} \psi_{2}^{2} .
\end{gathered}
$$


It follows from the Weingarten equations that

$$
(\partial-\mathcal{A})(\bar{\partial}-\mathcal{B}) \psi-(\bar{\partial}-\mathcal{B})(\partial-\mathcal{A}) \psi=\left(\mathcal{A}_{\bar{z}}-\mathcal{B}_{z}+[\mathcal{A}, \mathcal{B}]\right) \psi=0 .
$$

REMARK 2. In the cases $G=\mathbb{R}^{3}$ or $S U(2)$ the spinor $\psi^{*}=\left(-\bar{\psi}_{2}, \psi_{1}\right)^{\perp}$ meets the equation

$$
R \psi^{*}=\left(\mathcal{A}_{\bar{z}}-\mathcal{B}_{z}+[\mathcal{A}, \mathcal{B}]\right) \psi^{*}=0
$$

which together with $R \psi=0$ implies that

$$
R=\mathcal{A}_{\bar{z}}-\mathcal{B}_{z}+[\mathcal{A}, \mathcal{B}]=0
$$

(see [12]). In the case of $G=\mathrm{Nil}$ as well as for $G=\widetilde{S L}_{2}$ or Sol the equation $R \psi^{*}=0$ does not hold and, in particular, the kernel of the Dirac operator can not be treated as a vector space over quaternions. Therefore we shall derive the Codazzi equations by other methods.

We have

$$
\mathcal{A}=\left(\begin{array}{cc}
\alpha_{z}-\frac{i}{2} Z_{3} & A e^{-\alpha} \\
-W & -\frac{i}{2} Z_{3}
\end{array}\right), \quad \mathcal{B}=\left(\begin{array}{cc}
-\frac{i}{2} \bar{Z}_{3} & \bar{W} \\
-\bar{A} e^{-\alpha} & \alpha_{\bar{z}}-\frac{i}{2} \bar{Z}_{3}
\end{array}\right)
$$

where $Z_{3}=\psi_{1} \bar{\psi}_{2}$ and $W=\left(\frac{H}{2}-\frac{i}{4}\right) e^{\alpha}$. The equation $R \psi=0$ is written as the system of two equations:

$$
\begin{aligned}
& \kappa_{1}=\left(\left(\alpha_{z \bar{z}}-|A|^{2} e^{-2 \alpha}+|W|^{2}\right)+\frac{i}{2}\left(\partial \bar{Z}_{3}-\bar{\partial} Z_{3}\right)\right) \psi_{1}+\left(A_{\bar{z}} e^{-\alpha}-\bar{W}_{z}+\alpha_{z} \bar{W}\right) \psi_{2}=0, \\
& \kappa_{2}=\left(\bar{A}_{z} e^{-\alpha}-W_{\bar{z}}+\alpha_{\bar{z}} W\right) \psi_{1}+\left(-\left(\alpha_{z \bar{z}}-|A|^{2} e^{-2 \alpha}+|W|^{2}\right)+\frac{i}{2}\left(\partial \bar{Z}_{3}-\bar{\partial} Z_{3}\right)\right) \psi_{2}=0 .
\end{aligned}
$$

We recall that surfaces in Nil we have

$$
\partial \bar{Z}_{3}-\bar{\partial} Z_{3}=-\frac{i}{2}\left(\left|\psi_{2}\right|^{4}-\left|\psi_{1}\right|^{4}\right), \quad \partial \bar{Z}_{3}+\bar{\partial} Z_{3}=H\left(\left|\psi_{2}\right|^{4}-\left|\psi_{1}\right|^{4}\right) .
$$

The Codazzi equations is now the system

$$
\kappa_{1} \bar{\psi}_{1}-\bar{\kappa}_{2} \psi_{2}=0, \quad \kappa_{1} \bar{\psi}_{2}+\bar{\kappa}_{2} \psi_{1}=0
$$

which is rewritten as

$$
\begin{gathered}
\alpha_{z \bar{z}}-|A|^{2} e^{-2 \alpha}+\frac{H^{2}}{4} e^{2 \alpha}=\frac{1}{16}\left(3\left|\psi_{1}\right|^{4}+3\left|\psi_{2}\right|^{4}-10\left|\psi_{1}\right|^{2}\left|\psi_{2}\right|^{2}\right), \\
A_{\bar{z}}-\frac{H_{z}}{2} e^{2 \alpha}+\frac{1}{2}\left(\left|\psi_{2}\right|^{4}-\left|\psi_{1}\right|^{4}\right) \psi_{1} \bar{\psi}_{2}=0 .
\end{gathered}
$$

By using (22) we rewrite this system as follows

$$
\begin{array}{r}
\alpha_{z \bar{z}}-e^{-2 \alpha}|A|^{2}+\frac{1}{4} e^{2 \alpha} H^{2}=\frac{3}{16} e^{2 \alpha}-\left|Z_{3}\right|^{2}, \\
\bar{\partial}\left(A+\frac{Z_{3}{ }^{2}}{2 H+i}\right)=\frac{1}{2} H_{z} e^{2 \alpha}+\bar{\partial}\left(\frac{1}{2 H+i}\right) Z_{3}{ }^{2} .
\end{array}
$$

We summarize the above results in the following 
Theorem 1 For a surface in $G=$ Nil its generating spinor $\psi$ meets the Dirac equation (19).

Moreover each function $\psi$ meeting (19) serves as the generating spinor of a surface in Nil .

The Weingarten equations for the surface are given by (19) and (21). The Hopf differential takes the form (20) and the Codazzi equations are given by (23).

Corollary 1 The generating spinor $\psi$ of a minimal surface in Nil meets the following equations

$$
\bar{\partial} \psi_{1}=\frac{i}{4}\left(\left|\psi_{2}\right|^{2}-\left|\psi_{1}\right|^{2}\right) \psi_{1}, \quad \partial \psi_{2}=-\frac{i}{4}\left(\left|\psi_{2}\right|^{2}-\left|\psi_{1}\right|^{2}\right) \psi_{1} .
$$

Corollary 2 (Abresch) For a constant mean curvature surface in Nil the quadratic differential

$$
\widetilde{A} d z^{2}=\left(A+\frac{Z_{3}^{2}}{2 H+i}\right) d z^{2}
$$

is holomorphic.

Proposition 3 If the differential $\widetilde{A} d z^{2}$ is holomorphic then the surface in Nil has constant mean curvature.

Proof. We prove that by contradiction. Let us assume that $H_{z} \neq 0$ in some domain. Then we have

$$
\frac{1}{2} e^{2 \alpha}=\frac{2 H_{\bar{z}}}{(2 H+i)^{2}} Z_{3}^{2}
$$

and which implies the equality for the moduli of both sides:

$$
\frac{1}{2} e^{2 \alpha}=\frac{2\left|\psi_{1}\right|^{2}\left|\psi_{2}\right|^{2}}{4 H^{2}+1}
$$

Since $e^{\alpha}=\left|\psi_{1}\right|^{2}+\left|\psi_{2}\right|^{2}$, this is rewritten as

$$
\left(4 H^{2}+1\right)\left(\left|\psi_{1}\right|^{+}\left|\psi_{2}\right|^{2}\right)^{2}=4\left|\psi_{1}\right|^{2}\left|\psi_{2}\right|^{2}
$$

which implies

$$
4 H^{2} e^{2 \alpha}+\left(\left|\psi_{1}\right|^{2}-\left|\psi_{2}\right|^{2}\right)^{2}=0
$$

It is clear that this equality is valid if and only if $\left|\psi_{1}\right|=\left|\psi_{2}\right|$ and $H=0$, i.e. the surface is minimal. This contradiction proves the proposition.

Finally we compute the energy of a compact surface in Nil.

Proposition 4 Given a closed oriented surface $M$ in the group Nil, its energy is real-valued and equals

$$
E(M)=\int_{M}\left(\frac{H^{2}}{4}\left(\left|\psi_{1}\right|^{2}+\left|\psi_{2}\right|^{2}\right)^{2}-\frac{1}{16}\left(\left|\psi_{2}\right|^{2}-\left|\psi_{1}\right|^{2}\right)^{2}\right) \frac{i d z \wedge d \bar{z}}{2}
$$


Proof. Let us insert the formulas for $U_{\mathrm{Nil}}$ and $V_{\mathrm{Nil}}$ into (12) and obtain

$$
\bar{\partial}\left(\psi_{1} \bar{\psi}_{2}\right)=\frac{H}{2}\left(\left|\psi_{2}\right|^{2}-\left|\psi_{1}\right|^{2}\right)+i \frac{1}{4}\left(\left|\psi_{2}\right|^{4}-\left|\psi_{1}\right|^{4}\right) .
$$

By the Stokes formula we have

$$
\int_{M} \frac{H}{2}\left(\left|\psi_{2}\right|^{2}-\left|\psi_{1}\right|^{2}\right) \frac{i d z \wedge d \bar{z}}{2}+i \int_{M} \frac{1}{4}\left(\left|\psi_{2}\right|^{4}-\left|\psi_{1}\right|^{4}\right) \frac{i d z \wedge d \bar{z}}{2}=0 .
$$

The real part of the left hand side of this formula is a multiple of $\operatorname{Im} E(M)$ which implies that the energy is real-valued. We insert now $U_{\mathrm{Nil}}$ and $V_{\mathrm{Nil}}$ into $\operatorname{Re} E(M)$ and derive (24). This proves the proposition.

Notice that the vanishing of the the imaginary part of the left hand side of (25) together with (10) implies the equality

$$
\int_{M}\left\langle f^{-1}(N), e_{3}\right\rangle d \mu=\int_{M}\left(\left|\psi_{2}\right|^{4}-\left|\psi_{1}\right|^{4}\right) \frac{i d z \wedge d \bar{z}}{2}=0 .
$$

Proposition 5 The energy of a surface $M$ in the group Nil equals

$$
E(M)=\frac{1}{4} \int_{M}\left(H^{2}+\frac{\widehat{K}}{4}-\frac{1}{16}\right) d \mu
$$

where $\widehat{K}$ is the sectional curvature of the tangent plane at a point.

Proof. It is computed by using Proposition 2 that the sectional curvature of a tangent plane at the unit of group equals

$$
\widehat{K}=\frac{1}{4}-\cos ^{2} \varphi
$$

where $\varphi$ is the angle between the normal to the plane and $e_{3}$. By (10), the integrand in (24) is equal to

$$
\begin{gathered}
\frac{1}{4} e^{2 \alpha} H^{2}-\frac{1}{16}\left(\left|\psi_{2}\right|^{2}-\left|\psi_{1}\right|^{2}\right)^{2}=\frac{1}{4} e^{2 \alpha} H^{2}-\frac{1}{16} e^{2 \alpha}\left\langle f^{-1}(N), e_{3}\right\rangle^{2}= \\
\frac{1}{4} e^{2 \alpha}\left(H^{2}-\frac{1}{4} \cos ^{2} \varphi\right)=\frac{1}{4} e^{2 \alpha}\left(H^{2}-\frac{1}{4}\left(\frac{1}{4}-\widehat{K}\right)\right)= \\
=\frac{1}{4} e^{2 \alpha}\left(H^{2}+\frac{1}{4} \widehat{K}-\frac{1}{16}\right) .
\end{gathered}
$$

The proposition is proved. 


\subsection{The group $\widetilde{S L}_{2}$}

Inserting (14) into the derivational equations (6) and (7) we obtain

$$
\begin{aligned}
\partial \bar{Z}_{1}-\bar{\partial} Z_{1}+\frac{1}{2}\left(Z_{2} \bar{Z}_{3}-\bar{Z}_{2} Z_{3}\right)=0, \\
\partial \bar{Z}_{2}-\bar{\partial} Z_{2}+\frac{1}{2}\left(Z_{3} \bar{Z}_{1}-\bar{Z}_{3} Z_{1}\right)=0, \\
\partial \bar{Z}_{3}-\bar{\partial} Z_{3}-2\left(Z_{1} \bar{Z}_{2}-\bar{Z}_{1} Z_{2}\right)=0, \\
\partial \bar{Z}_{1}+\bar{\partial} Z_{1}-\frac{5}{2}\left(Z_{2} \bar{Z}_{3}+\bar{Z}_{2} Z_{3}\right)=2 i H\left(\bar{Z}_{2} Z_{3}-Z_{2} \bar{Z}_{3}\right), \\
\partial \bar{Z}_{2}+\bar{\partial} Z_{2}+\frac{5}{2}\left(Z_{1} \bar{Z}_{3}+\bar{Z}_{1} Z_{3}\right)=2 i H\left(\bar{Z}_{3} Z_{1}-Z_{3} \bar{Z}_{1}\right), \\
\partial \bar{Z}_{3}+\bar{\partial} Z_{3}=2 i H\left(\bar{Z}_{1} Z_{2}-Z_{1} \bar{Z}_{2}\right) .
\end{aligned}
$$

By inserting (8) into these equations we reduce the system of the first two of them to

$$
\partial \psi_{2}^{2}+\bar{\partial} \psi_{1}^{2}-\frac{i}{2} \psi_{1} \psi_{2}\left(\left|\psi_{1}\right|^{2}+\left|\psi_{2}\right|^{2}\right)=0
$$

and the fourth and fifth equations together are reduced to

$$
\partial \psi_{2}^{2}-\bar{\partial} \psi_{1}^{2}+\frac{5}{2} i \psi_{1} \psi_{2}\left(\left|\psi_{1}\right|^{2}-\left|\psi_{2}\right|^{2}\right)=-2 H \psi_{1} \psi_{2}\left(\left|\psi_{1}\right|^{2}+\left|\psi_{2}\right|^{2}\right) .
$$

These two equations are written in terms of the Dirac equation (the derivation is completely similar to the Nil case, see (19) in 3.2 :

$$
\begin{gathered}
\mathcal{D}_{\mathrm{SL}} \psi=\left[\left(\begin{array}{cc}
0 & \partial \\
-\bar{\partial} & 0
\end{array}\right)+\left(\begin{array}{cc}
U_{\mathrm{SL}} & 0 \\
0 & V_{\mathrm{SL}}
\end{array}\right)\right] \psi=0, \\
U_{\mathrm{SL}}=\frac{H}{2}\left(\left|\psi_{1}\right|^{2}+\left|\psi_{2}\right|^{2}\right)+i\left(\frac{1}{2}\left|\psi_{1}\right|^{2}-\frac{3}{4}\left|\psi_{2}\right|^{2}\right), \\
V_{\mathrm{SL}}=\frac{H}{2}\left(\left|\psi_{1}\right|^{2}+\left|\psi_{2}\right|^{2}\right)+i\left(\frac{3}{4}\left|\psi_{1}\right|^{2}-\frac{1}{2}\left|\psi_{2}\right|^{2}\right),
\end{gathered}
$$

where $H$ is the mean curvature of the surface.

The Hopf differential equals

$$
A=\left(\bar{\psi}_{2} \partial \psi_{1}-\psi_{1} \partial \bar{\psi}_{2}\right)-\frac{5 i}{2} \psi_{1}^{2} \bar{\psi}_{2}^{2}
$$

and we have

$$
\begin{gathered}
\partial \psi_{1}=\alpha_{z} \psi_{1}+A e^{-\alpha} \psi_{2}+\frac{5 i}{4} \psi_{1}^{2} \bar{\psi}_{2}, \\
\bar{\partial} \psi_{2}=-\bar{A} e^{-\alpha} \psi_{1}+\alpha_{\bar{z}} \psi_{2}+\frac{5 i}{4} \bar{\psi}_{1} \psi_{2}^{2}
\end{gathered}
$$

The matrices $\mathcal{A}$ and $\mathcal{B}$ are given by the formulas

$$
\mathcal{A}=\left(\begin{array}{cc}
\alpha_{z}+\frac{5 i}{4} Z_{3} & A e^{-\alpha} \\
-W & \frac{5 i}{4} Z_{3}
\end{array}\right), \quad \mathcal{B}=\left(\begin{array}{cc}
\frac{5 i}{4} \bar{Z}_{3} & \bar{W} \\
-\bar{A} e^{-\alpha} & \alpha_{\bar{z}}+\frac{5 i}{4} \bar{Z}_{3}
\end{array}\right)
$$


where $W=\frac{1}{2}(H+i) e^{\alpha}$. The equation $R \psi=0$ is equivalent to the equations $\kappa_{1}=\left(\left(\alpha_{z \bar{z}}-|A|^{2} e^{-2 \alpha}+|W|^{2}\right)-\frac{5 i}{4}\left(\partial \bar{Z}_{3}-\bar{\partial} Z_{3}\right)\right) \psi_{1}+\left(A_{\bar{z}} e^{-\alpha}-\bar{W}_{z}+\alpha_{z} \bar{W}\right) \psi_{2}=0$, $\kappa_{2}=\left(\bar{A}_{z} e^{-\alpha}-W_{\bar{z}}+\alpha_{\bar{z}} W\right) \psi_{1}+\left(-\left(\alpha_{z \bar{z}}-|A|^{2} e^{-2 \alpha}+|W|^{2}\right)-\frac{5 i}{4}\left(\partial \bar{Z}_{3}-\bar{\partial} Z_{3}\right)\right) \psi_{2}=0$. Since the generating spinors of surfaces in $\widetilde{S L}_{2}$ meet the equalities

$$
\partial \bar{Z}_{3}-\bar{\partial} Z_{3}=i\left(\left|\psi_{2}\right|^{4}-\left|\psi_{1}\right|^{4}\right), \quad \partial \bar{Z}_{3}+\bar{\partial} Z_{3}=H\left(\left|\psi_{2}\right|^{4}-\left|\psi_{1}\right|^{4}\right) .
$$

we rewrite the equations $\kappa_{1} \bar{\psi}_{1}-\bar{\kappa}_{2} \psi_{2}=0$ and $\kappa_{1} \bar{\psi}_{2}+\bar{\kappa}_{2} \psi_{1}=0$ as the following system

$$
\begin{array}{r}
\alpha_{z \bar{z}}-e^{-2 \alpha}|A|^{2}+\frac{1}{4} e^{2 \alpha} H^{2}=e^{2 \alpha}-5\left|Z_{3}\right|^{2}, \\
\bar{\partial}\left(A+\frac{5 Z_{3}^{2}}{2(H-i)}\right)=\frac{1}{2} H_{z} e^{2 \alpha}+\bar{\partial}\left(\frac{5}{2(H-i)}\right) Z_{3}{ }^{2} .
\end{array}
$$

We derive

Theorem 2 Given a surface in $G=\widetilde{S L}_{2}$, its generating spinor $\psi$ satisfies the Dirac equation (28).

Any function $\psi$ meeting (28) is the generating spinor of a surface in $\widetilde{S L}$.

The Weingarten equations for the surface is the system consisting of (28) and (30). The Hopf differential is given by (20) and the Codazzi equations take the form (23).

Corollary 3 The generating spinor of a minimal surface in $\widetilde{S L}_{2}$ satisfies the equations

$$
\bar{\partial} \psi_{1}=i\left(\frac{3}{4}\left|\psi_{1}\right|^{2}-\frac{1}{2}\left|\psi_{2}\right|^{2}\right) \psi_{2}, \quad \partial \psi_{2}=-i\left(\frac{1}{2}\left|\psi_{1}\right|^{2}-\frac{3}{4}\left|\psi_{2}\right|^{2}\right) \psi_{1} .
$$

Corollary 4 (Abresch) For a constant mean curvature surface in $\widetilde{S L}_{2}$ the quadratic differential

$$
\widetilde{A} d z^{2}=\left(A+\frac{5}{2(H-i)} Z_{3}^{2}\right) d z^{2}
$$

is holomorphic.

REMARK 3. The method of proving Proposition 3 fails in the case of $\widetilde{S L}_{2}$ and we do not know whether the holomorphicity of $\widetilde{A}$ implies that the surface has constant mean curvature.

We finish our study of surfaces in $\widetilde{S L}_{2}$ by computing the energy functional. 
Proposition 6 Given a closed oriented surface $M$ in the group $\widetilde{S L}_{2}$, its energy is real-valued and equals

$$
\begin{array}{r}
E(M)=\int_{M}\left[\frac{H^{2}}{4}\left(\left|\psi_{1}\right|^{2}+\left|\psi_{2}\right|^{2}\right)^{2}-\right. \\
\left.\left(\frac{1}{2}\left|\psi_{1}\right|^{2}-\frac{3}{4}\left|\psi_{2}\right|^{2}\right)\left(\frac{3}{4}\left|\psi_{1}\right|^{2}-\frac{1}{2}\left|\psi_{2}\right|^{2}\right)\right] \frac{i d z \wedge d \bar{z}}{2} .
\end{array}
$$

The proof of this proposition is straightforward and analogous to the proof of Proposition 4 Hence we skip it and only mention that as for surfaces in Nil we have $\int_{M}\left\langle f^{-1}(N), e_{3}\right\rangle d \mu=\int_{M}\left(\left|\psi_{2}\right|^{4}-\left|\psi_{1}\right|^{4}\right) \frac{i d z \wedge d \bar{z}}{2}=0$.

As for the group Nil we can write down the formula for the energy in common geometric terms.

Proposition 7 The energy of a surface $M \subset \widetilde{S L}_{2}$ equals

$$
E(M)=\frac{1}{4} \int_{M}\left(H^{2}+\frac{5}{16} \widehat{K}-\frac{1}{4}\right) d \mu,
$$

where $\widehat{K}$ is the sectional curvature of the tangent plane at a point.

This proposition is straightforward from (24) and Proposition 2

\subsection{The group Sol}

By (15), the derivational equations (6) and (7) for surfaces in the group Sol take the form

$$
\begin{array}{r}
\partial \bar{Z}_{1}-\bar{\partial} Z_{1}+\left(Z_{1} \bar{Z}_{3}-\bar{Z}_{1} Z_{3}\right)=0, \\
\partial \bar{Z}_{2}-\bar{\partial} Z_{2}=\left(Z_{2} \bar{Z}_{3}-\bar{Z}_{2} Z_{3}\right)=0, \\
\partial \bar{Z}_{3}-\bar{\partial} Z_{3}=0, \\
\partial \bar{Z}_{1}+\bar{\partial} Z_{1}+\left(Z_{1} \bar{Z}_{3}+\bar{Z}_{1} Z_{3}\right)=2 i H\left(\bar{Z}_{2} Z_{3}-Z_{2} \bar{Z}_{3}\right), \\
\partial \bar{Z}_{2}+\bar{\partial} Z_{2}-\left(Z_{2} \bar{Z}_{3}+\bar{Z}_{2} Z_{3}\right)=2 i H\left(\bar{Z}_{3} Z_{1}-Z_{3} \bar{Z}_{1}\right), \\
\partial \bar{Z}_{3}+\bar{\partial} Z_{3}-2\left(\left|Z_{1}\right|^{2}-\left|Z_{2}\right|^{2}\right)=2 i H\left(\bar{Z}_{1} Z_{2}-Z_{1} \bar{Z}_{2}\right) .
\end{array}
$$

As in 3.2 and $\$ 3.3$ we insert (8) in these equations and derive the following pair of equations

$$
\begin{gathered}
\partial \psi_{2}^{2}+\bar{\partial} \psi_{1}^{2}-\bar{\psi}_{1} \bar{\psi}_{2}\left(\left|\psi_{1}\right|^{2}+\left|\psi_{2}\right|^{2}\right)=0 \\
\partial \psi_{2}^{2}-\bar{\partial} \psi_{1}^{2}+\bar{\psi}_{1} \bar{\psi}_{2}\left(\left|\psi_{1}\right|^{2}-\left|\psi_{2}\right|^{2}\right)=-2 H \psi_{1} \psi_{2}\left(\left|\psi_{1}\right|^{2}+\left|\psi_{2}\right|^{2}\right) .
\end{gathered}
$$

We see that at points where $Z_{3}=\psi_{1} \bar{\psi}_{2} \neq 0$ these equations are rewritten in terms of the Dirac equation

$$
\begin{array}{r}
\mathcal{D}_{\text {Sol }} \psi=\left[\left(\begin{array}{cc}
0 & \partial \\
-\bar{\partial} & 0
\end{array}\right)+\left(\begin{array}{cc}
U_{\text {Sol }} & 0 \\
0 & V_{\text {Sol }}
\end{array}\right)\right] \psi=0, \\
U_{\text {Sol }}=\frac{H}{2}\left(\left|\psi_{1}\right|^{2}+\left|\psi_{2}\right|^{2}\right)+\frac{1}{2} \bar{\psi}_{2}^{2} \frac{\bar{\psi}_{1}}{\psi_{1}}, \\
V_{\text {Sol }}=\frac{H}{2}\left(\left|\psi_{1}\right|^{2}+\left|\psi_{2}\right|^{2}\right)+\frac{1}{2} \bar{\psi}_{1}^{2} \frac{\bar{\psi}_{2}}{\psi_{2}} .
\end{array}
$$


REMARK 4. Since the left invariant vector fields generated by $e_{1}$ and $e_{2}$ commute the equation $Z_{3}=\psi_{1} \bar{\psi}_{2}=0$ can be valid in an open subset $B$ of a surface. Therefore the Dirac equation can not be extended by continuity onto the whole surface and does not describe $\psi$ in $B$. Since $H=0$ in $B$ it is reasonable to assume that $U_{\text {Sol }}=V_{\text {Sol }}=0$ inside such a domain. However the potentials $U_{\text {Sol }}$ and $V_{\text {Sol }}$ are not always correctly defined on the boundaries of the set $\left\{Z_{3} \neq 0\right\}$ in view of the indeterminacy of $\frac{\bar{\psi}_{1}}{\psi_{1}}$ and $\frac{\bar{\psi}_{2}}{\psi_{2}}$. The measure of the indeterminacy set is zero and we may correctly define the energy of a surface in Sol as

$$
E(M)=\int_{\left\{Z_{3} \neq 0\right\}} U_{\text {Sol }} V_{\text {Sol }} d \mu .
$$

We did not manage to rewrite this quantity in common geometric terms as we did for surfaces in Nil and $\widetilde{S L}_{2}$ and its geometric meaning remains still unclear for us. We even do not know whether the energy real-valued (as for surfaces in $S U(2)$, Nil, and $\left.\widetilde{S L}_{2}\right)$ or not.

The Hopf differential of a surface in Sol equals

$$
A=\left(\bar{\psi}_{2} \partial \psi_{1}-\psi_{1} \partial \bar{\psi}_{2}\right)+\frac{1}{2}\left(\bar{\psi}_{2}^{4}-\psi_{1}^{4}\right)
$$

and we complete the Weingarten equations by the following system

$$
\begin{gathered}
\partial \psi_{1}=\alpha_{z} \psi_{1}+A e^{-\alpha} \psi_{2}-\frac{1}{2} \bar{\psi}_{2}^{3}, \\
\bar{\partial} \psi_{2}=-\bar{A} e^{-\alpha} \psi_{1}+\alpha_{\bar{z}} \psi_{2}-\frac{1}{2} \bar{\psi}_{1}^{3} .
\end{gathered}
$$

The Codazzi equations take the form

$$
\begin{array}{r}
\alpha_{z \bar{z}}-e^{-2 \alpha}|A|^{2}+\frac{1}{4} e^{2 \alpha} H^{2}=\frac{1}{4}\left(6\left|\psi_{1}\right|^{2}\left|\psi_{2}\right|^{2}-\left(\left|\psi_{1}\right|^{4}+\left|\psi_{2}\right|^{4}\right)\right), \\
A_{\bar{z}}-\frac{1}{2} H_{z} e^{2 \alpha}=\left(\left|\psi_{2}\right|^{4}-\left|\psi_{1}\right|^{4}\right) \psi_{1} \bar{\psi}_{2} .
\end{array}
$$

Let us summarize these results. Let $f: M \rightarrow$ Sol be a surface. We by $B$ the subset of $M$ where $Z_{3}=\left\langle f^{-1} f_{z}, e_{3}\right\rangle=0$, by $B_{0}$ the interior of $B$, and by $C$ the subset of $M$ where $Z_{3} \neq 0$. Then $M=B_{0} \cup \bar{C}$ and the set $B \backslash B_{0}$ lies in the closure $\bar{C}$ of $C$ and has measure zero.

Theorem 3 The generating spinor $\psi$ of a surface $f: M \rightarrow$ Sol meets the Dirac equation (35) in $C$ and meets the Dirac equation with zero potentials: $\bar{\partial} \psi_{1}=\partial \psi_{2}=0$, in $B_{0}$.

Every function $\psi$ meeting (35) is some set $D \subset M$ is the generating function of a surface $f: D \rightarrow$ Sol.

The Hopf differential is given by (36). In $B_{0} \cup C$ the Weingarten equations are formed by (37) and by the Dirac equation for $\psi$. The Codazzi equations take the form (38). 
Corollary 5 The generating spinor $\psi$ of a minimal surface in Sol meets the equations

$$
\bar{\partial} \psi_{1}=\frac{1}{2} \bar{\psi}_{1}^{2} \bar{\psi}_{2}, \quad \partial \psi_{2}=-\frac{1}{2} \bar{\psi}_{1} \bar{\psi}_{2}^{2}
$$

Addition to the proofs. After submitting this paper to the journal we became aware of the papers [14, 15, where the authors obtained some interesting results on minimal surfaces in three-dimensional Lie groups under study. In particular, some analogs of the Weierstrass representation for minimal surfaces in Nil and Sol were derived.

\section{References}

[1] Abresch, U.: Generalized Hopf differentials, to appear in Proceedings of the 13th School of Differential geometry, Mat. Contemp., 2004.

[2] Abresch, U., and Rosenberg, H.: The Hopf differential for constant mean curvature surfaces in $S^{2} \times \mathbb{R}$ and $H^{2} \times \mathbb{R}$, Acta Mathematica 193 (2004), $141-174$.

[3] Daniel, B.: Isometric immersions into 3-dimensional homogeneous manifolds, arxiv: math.DG/0503500

[4] Figueroa, C., Mercuri, F., and Pedrosa, R.: Invariant surfaces of the Heisenberg groups, Ann. Math. Pura Appl. 177 (1999), 173-194.

[5] Fokas, A.S., and Gelfand, I.M.: Surfaces on Lie groups, on Lie algebras, and their integrability. Comm. Math. Phys. 177 (1996), 203-220.

[6] Haskins, M.: The geometric complexity of special Lagrangian $T^{2}$-cones, arxiv: math.DG/0307129

[7] Hitchin, N.: Harmonic maps from a 2-torus to the 3-sphere. J. Differ. Geom. 31 (1990), 627-710.

[8] Milnor, J.W.: Curvatures of left invariant metrics on Lie groups. Adv. Math. 21 (1976), 293-329.

[9] Scott, P.: The geometries of 3-manifolds. Bull. London Math. Soc. 56 (1983), 401-487.

[10] Taimanov, I.A.: Modified Novikov-Veselov equation and differential geometry of surfaces. Amer. Math. Soc. Transl., Ser. 2, V. 179, 1997, pp. $133-151$.

[11] Taimanov, I. A.: The Weierstrass representation of closed surfaces in $\mathbb{R}^{3}$. Functional Anal. Appl. 32:4 (1998), 49-62.

[12] Taimanov, I.A.: Dirac operators and conformal invariants of tori in 3-space. Proc. Steklov Inst. Math. 244 (2004), 233-263. 
[13] Thurston, W.P.: Three dimensional manifolds, Kleinian groups and hyperbolic geometry. Bull. Amer. Math. Soc. 6 (1982), 357-381.

[14] Inoguchi, J., Kumamoto, T., Ohsugi, N., Suyama, Y.: Differential geometry of curves and surfaces in 3-dimensional homogeneous spaces.II. Fukuoka University Science Reports 30 (2000), 17-47.

[15] Inoguchi, J.: Minimal surfaces in 3-dimensional solvable Lie groups. Chin. Ann. Math. 24B:1 (2003), 73-84. 